\title{
Freeflow-2D - Um Sistema de Simulação de Escoamentos de Fluidos com Superfícies Livres Bidimensionais
}

J. de OLIVEIRA, A. CASTELO FILHO, Departamento de Ciências de Computação e Estatística, Instituto de Ciências Matemáticas e de Computação, USP, Cx.P. 668, 13560-970 São Carlos, SP, Brasil.

\begin{abstract}
Resumo. Este trabalho apresenta um ambiente integrado para simulação de escoamentos bidimensionais incompressíveis com superfícies livres, denominado Freeflow2D. Este sistema é composto por quatro módulos: um modelador de moldes e escoamentos - Modflow-2D, um simulador de escoamentos - Simflow-2D, um visualizador de escoamentos - Visflow-2D e um reiniciador de escoamentos - Resimflow-2D. A comunicação entre os módulos do sistema é feita por arquivos. O Freeflow-2D implementa o método GENSMAC e foi baseado na estrutura de dados do Freeflow-3D. Os objetos geométricos (fluidos, contêineres, injetores e ejetores) são representados pela estrutura de dados B-Rep (Boundary Representation).
\end{abstract}

\section{Introdução}

A modelagem e simulação de muitos problemas em mecânica dos fluidos tornou-se possível devido não só ao avanço da tecnologia computacional, como também das técnicas numéricas.

Nas últimas três décadas muitos problemas em dinâmica dos fluidos, como o escoamento de fluidos com superfícies livres, têm sido estudados. Como exemplo de aplicação, tem-se o preenchimento de cavidades (indústria alimentícia) e injeção em moldes (indústria siderúrgica e de plástico).

Muitos pesquisadores têm obtido sucesso ao tratar escoamentos de fluidos com superfícies livres utilizando o método de diferenças finitas. O método MAC (Markerand-Cell) [1], introduzido por Harlow e Welch, foi uma das primeiras técnicas a resolver satisfatoriamente problemas com superfícies livres. Este método utiliza partículas virtuais que representam o fluido, cujas coordenadas são armazenadas e movem-se de uma célula a outra de acordo com a última velocidade calculada, se a célula contém uma partícula considera-se que contém fluido, assim fornecendo a visualização da superfície livre.

Em seguida, Amsden e Harlow desenvolveram o método SMAC [2] (que é o método MAC Simplificado) o qual divide os ciclos de cálculos em duas partes: velocidade e pressão, não existindo, assim, processo iterativo envolvendo velocidade e pressão, evitando-se algumas dificuldades encontradas no método MAC. 
Em 1994, Tomé e McKee desenvolveram o método GENSMAC [3, 4], baseandose no método SMAC, para escoamentos bidimensionais de fluidos newtonianos incompressíveis com superfícies livres em domínios complexos. Posteriormente, o método GENSMAC foi expandido para tratar fluidos não newtonianos.

Baseado no método GENSMAC, foi desenvolvido um ambiente de modelagem, simulação e visualização de escoamentos bidimensionais incompressíveis com superfícies livres denominado Freeflow [5], mas este sistema possui uma estrutura de dados de difícil extensão.

Por existir muitos problemas originalmente tridimensionais foi desenvolvido no Instituto de Ciências Matemáticas e de Computação - ICMC, o sistema Freeflow-3D [6], um sistema de modelagem, simulação e visualização de escoamento de fluidos com superfícies livres tridimensionais. Neste sistema, é implementado o método GENSMAC-3D [7], que é uma extensão do método GENSMAC.

$\mathrm{O}$ projeto aqui descrito objetivou investigar estes sistemas e assuntos correlatos, para a implementação de ambientes de simulação bi e tridimensional integrados. Com isto, foi desenvolvido o ambiente de simulação Freeflow-2D [8], um ambiente integrado para simulação de escoamentos bidimensionais incompressíveis com superfícies livres. O Freeflow-2D basea-se no método GENSMAC e na estrutura de dados do Freeflow-3D.

A organização deste trabalho é feita da seguinte maneira: na seção 2 é dado uma breve descrição do Freeflow-2D, na seção 3 descreve-se a estrutura de dados criada para o Freeflow-2D, nas seções 4, 5, 6 e 7 detalha-se os módulos do Freeflow-2D, modelador, simulador, visualizador e reiniciador, respectivamente. Na seção 8 é exemplificado a comunicação entre os módulos, na seção 9 tem-se alguns resultados de simulações realizadas pelo Freeflow-2D e, por fim, na seção 10 tem-se as conclusões e contribuições deste trabalho.

\section{Freeflow-2D}

O Freeflow-2D é um sistema integrado para modelagem, simulação e visualização de escoamentos bidimensionais incompressíveis com superfícies livres.

O sistema Freeflow-2D contém quatro módulos:

- Modflow-2D (modelador de moldes e escoamentos): um sistema interativo para a especificação de um modelo de escoamento de fluidos que inclui a definição de elementos no domínio do escoamento como moldes, injetores, fluidos e a definição de propriedades do escoamento;

- Simflow-2D (simulador de escoamentos): um sistema para simulação de escoamentos que é a parte central do Freeflow-2D, pois implementa a discretização das equações governantes (o simulador usa aproximação por diferenças finitas para resolver as equações de Navier-Stokes), assim como as condições de fronteira. $\mathrm{O}$ método GENSMAC serviu de base para a implementação do simulador;

- Visflow-2D (visualizador de escoamentos): um sistema interativo para a tradução 
dos resultados produzidos pelo simulador em imagens de fácil interpretação;

- Resimflow-2D (reiniciador de escoamentos): um sistema interativo que faz a alteração de alguns parâmetros durante a simulação.

A implementação dos quatro módulos que compõem o sistema Freeflow-2D foi feita utilizando a linguagem $\mathrm{C}$ sobre o sistema operacional UNIX. As interfaces gráficas dos módulos Modflow-2D, Visflow-2D e Resimflow-2D utilizam o sistema de gerenciamento de janelas Xview sobre o Xwindows. A estrutura de dados adotada proporciona acesso fácil às informações e possui independência dos dados de forma a simplificar a manutenção e extensão do código. Os programas foram desenvolvidos em estações de trabalho Sun Solaris, com versões executando em ambientes LinuxPC. A comunicação entre os módulos do sistema é feita através de arquivos.

\section{Estrutura de Dados}

A estrutura de dados utilizada no Freeflow-2D oferece acesso fácil às informações e possui independência dos dados de forma a simplificar a manutenção e extensão do código. Foi baseada na estrutura de dados do Freeflow-3D para que, no futuro, os ambientes de simulação bi e tridimensionais sejam integrados.

Os objetos geométricos no Freeflow-2D são curvas fechadas com orientação antihorária e são representados por um tipo de estrutura de dados B-Rep (Boundary Representation) para representar objetos geométricos pela sua fronteira (faces, arestas, vértices e suas relações topológicas). A estrutura de dados utilizada no Freeflow-2D foi denominada de halfedge-2d. A estrutura halfedge-2d é uma variação da estrutura de dados half-edge utilizada no Freeflow-3D. Os níveis hierárquicos da estrutura halfedge-2d estão representados na Figura 1.

As listas fatia, face e vértice são abertas, e a lista semi-aresta é fechada. Cada elemento fatia aponta para as listas de faces e vértices. Cada elemento face aponta para a fatia e para a lista de semi-aresta. Cada semi-aresta aponta para uma face e para um vértice e, finalmente, cada vértice aponta para uma semi-aresta.

Como no Freeflow-3D, os dados no Freeflow-2D estão divididos em duas categorias:

- Dados diretos: domínio, velocidade, pressão, células, parâmetros utilizados pelo simulador e representação dos objetos geométricos do modelo. Os dados diretos estão subdivididos em:

- dados estáticos (não são modificados durante a simulação): domínio, discretização, parâmetros de adimensionalização, algumas propriedades do escoamento e do fluido (viscosidade e o campo de gravidade);

- dados dinâmicos (são os que se modificam durante a simulação): velocidade, pressão configuração do conjunto de células, representação dos objetos geométricos.

Os dados dinâmicos que são representados por matrizes são: velocidade e pres- 


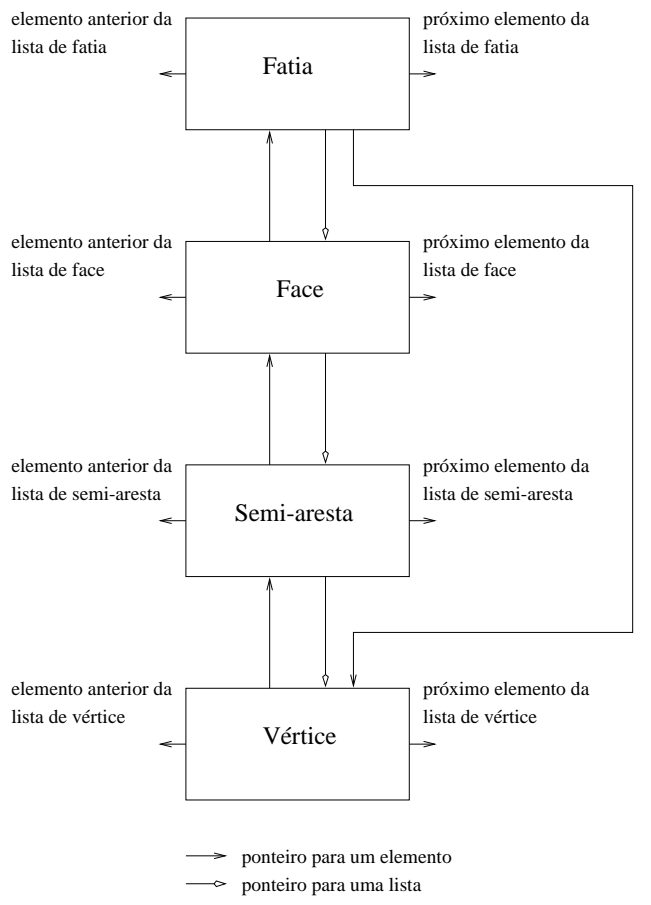

Figura 1: Níveis hierárquicos da estrutura de dados

são. A configuração do conjunto de células é representada por matrizes e árvores. Utiliza-se estes dois tipos de representação pela necessidade de acesso a um determinado grupo específico de células e, para isso, utiliza-se a representação por árvore. No caso de acesso a uma vizinhança de uma determinada célula, a representação matricial é mais adequada;

- Dados indiretos: esta estrutura foi desenvolvida preocupando-se com a representação dos objetos por sua fronteira, o desempenho dos algoritmos envolvidos no simulador e a minimização da interdependência entre dados. Embora o acesso aos dados da estrutura halfedge-2d que representa os contêineres, injetores, ejetores e fluidos seja direto, o acesso a um determinado objeto não é feito diretamente. Estes dados são compostos por três estruturas:

- contêiner (representa os contêineres): é composta de dados geométricos (BRep) do contêiner, dados geométricos do ejetor (caso exista), tipos de condição de contorno e uma árvore que armazena informações sobre as células que definem esse contêiner e atributos específicos do contêiner representado;

- injetor (representa os injetores): é composta de dados geométricos (B-Rep), características do injetor, informações sobre os contêineres e fluidos que relacionam- 
se com esse injetor, uma árvore que armazena informações sobre as células que o definem e atributos específicos;

- ejetor (representa os ejetores): é composta de dados geométricos (B-Rep), tipos de condições de contorno, informações sobre o contêiner que o contém, uma árvore que armazena informações sobre células que definem esse ejetor e atributos específicos.

\section{Modelador}

O Modflow-2D é o módulo responsável pela introdução de dados que caracterizam o escoamento a ser simulado e possibilita a definição de elementos no domínio do escoamento. O modelador possui uma interface gráfica de fácil execução e requer a introdução de informações do domínio e da configuração do escoamento para a sua execução.

Os dados que configuram o escoamento são: domínio, células, dimensão das células, tempo inicial e final, ciclo inicial e final, espaçamento de tempo para impressão e para gravação automática, escala de comprimento e velocidade, força de gravidade (nos eixos x e y), viscosidade, incremento de tempo inicial, tolerância para a solução da equação de Poisson, fatores de controle do passo, opções de escolha do método para solução das equações de velocidades entre explícito e implícito (está implementado somente o explícito) e tipo de escoamento entre Newtoniano, Cross-Model e Power-Law (só está implementado o Newtoniano). Para todas as configurações tem-se dados padrão de entrada (default).

Este módulo também permite a visualização dos dados geométricos gerados, além das operações geométricas usuais, como rotações, translações e ampliações. Outra facilidade é a possibilidade de alterar propriedades de visualização dos objetos, como cor e visibilidade.

\section{Simulador}

O simulador consiste de um conjunto de programas baseado no método GENSMAC, cuja finalidade é resolver problemas de escoamentos transientes de fluidos newtonianos incompressíveis com superfícies livres. O Simflow-2D representa o fluido por partículas apenas na fronteira utilizando a estrutura halfedge-2d.

Este módulo resolve as equações de Navier-Stokes (equação de momento e da continuidade) em duas dimensões para um fluido incompressível:

$$
\begin{gathered}
\rho \frac{D \boldsymbol{u}}{D t}=\nabla . \boldsymbol{\sigma}+\rho \boldsymbol{g}, \quad \text { equação de momento, } \\
\nabla \cdot \boldsymbol{u}=0 \quad \text { equação da continuidade, }
\end{gathered}
$$

onde $\rho$ é a densidade, $\boldsymbol{u}(\boldsymbol{x}, t)$ a velocidade ( $\boldsymbol{x}$ é a posição no espaço e $t$ é o tempo), $g$ o campo gravitacional e 


$$
\boldsymbol{\sigma}=-p I+\boldsymbol{\tau}
$$

a tensão, sendo que $p$ é a pressão, $I$ a matriz identidade e $\tau$ o tensor extra tensão.

O tensor $\boldsymbol{\tau}$ é dado pela equação:

$$
\boldsymbol{\tau}=2 \mu(q) \boldsymbol{d}
$$

onde $\mu(q)$ é a viscosidade aparente dependente da razão de cisalhamento local

$$
q=\left(2 \operatorname{tr}\left(d^{2}\right)\right)^{\frac{1}{2}}
$$

e

$$
\boldsymbol{d}=\frac{1}{2}\left[(\nabla \boldsymbol{u})+(\nabla \boldsymbol{u})^{t}\right]
$$

é o tensor razão de deformação. A viscosidade $\mu=\rho \nu$, onde $\nu$ é a viscosidade cinemática $\left(\frac{\mu}{\rho}\right)$.

$\mathrm{Na}$ fronteira livre as condições de contorno para a pressão e velocidade são dadas pelas relações: $\boldsymbol{n} .(\boldsymbol{\sigma} \cdot \boldsymbol{n})=0$ e $\boldsymbol{m} .(\boldsymbol{\sigma} . \boldsymbol{n})=0$, onde $\boldsymbol{n}=\left(n_{x}, n_{y}\right)$ e $\boldsymbol{m}=\left(-n_{y}, n_{x}\right)$ denotam vetor normal unitário e vetor tangente, respectivamente, à superfície.

Com partículas marcadoras usadas para representar o fluido, as equações são resolvidas em uma rede alternada para o tempo presente. Mais precisamente, o procedimento pode ser exposto como segue. Suponha que no tempo dado $t_{0}$, o campo de velocidade é conhecido e as condições de fronteira para a velocidade e pressão são dadas. O campo de velocidade atualizado $\boldsymbol{u}(\boldsymbol{x}, t)$, onde $t=t_{0}+\delta t$, é então calculado como segue:

(i) Através de $\boldsymbol{u}\left(\boldsymbol{x}, t_{0}\right)$, calcula-se $\boldsymbol{q}\left(\boldsymbol{x}, t_{0}\right)$ e $\nu\left(q\left(\boldsymbol{x}, t_{0}\right)\right)$;

(ii) Seja $\tilde{p}\left(\boldsymbol{x}, t_{0}\right)$ uma pressão arbitrária;

(iii) Calcula-se um campo de velocidade intermediária, $\tilde{\boldsymbol{u}}(x, t)$, através de $\frac{\partial \tilde{\boldsymbol{u}}}{\partial t}=$ $-\nabla \tilde{p}+N(\boldsymbol{u})$, onde $N(\boldsymbol{u})=-\nabla \tau+\rho \boldsymbol{g} ;$

(iv) Resolve-se a equação de Poisson $\nabla^{2} \psi(\boldsymbol{x}, t)=\nabla \cdot \tilde{\boldsymbol{u}}(\boldsymbol{x}, t)$ com as condições de fronteira adequadas. Elas são $\psi=0$ (condição homogênea de Dirichlet na superfície livre) e $\frac{\partial \psi}{\partial \boldsymbol{n}}=0$ (condição homogênea de Newmann na fronteira rígida);

(v) Calcula-se a velocidade $\boldsymbol{u}(\boldsymbol{x}, t)=\tilde{\boldsymbol{u}}(\boldsymbol{x}, t)-\nabla \psi(\boldsymbol{x}, t)$;

(vi) Cálcula-se a pressão $\frac{\partial}{\partial t}(\boldsymbol{u}-\tilde{\boldsymbol{u}})=-\nabla(p-\tilde{p})$;

(vii) E, por fim, atualiza-se as posições das partículas marcadoras que constitui-se de três etapas:

- por $\frac{d x}{d t}=u$ e $\frac{d y}{d t}=v$ se tem a nova posição das partículas virtuais;

- inserir partículas (representadas pelos vértices da estrutura halfedge-2d): 
- para se inserir partículas no fluido considera-se o comprimento de cada aresta que representa a superfície livre, subdividindo essa aresta em arestas menores toda vez que seu comprimento for superior a $0.8 * \min \{d x, d y\}$.

O processo de inserção é importante para que o sistema funcione bem, pois conforme o fluido se move as partículas que representam a superfície podem ficar distantes uma das outras e assim perde-se os detalhes da representação. Pode ocorrer também que uma célula da superfície livre não contenha partículas devido a definição das células ser feita no instante de tempo anterior, podendo haver confusão no algoritmo de definição das células no tempo atualizado;

- eliminar partículas: é importante para diminuição dos dados e faz-se quando uma aresta contém vértices numa mesma célula e seu tamanho é inferior a $0.05 *$ $\min \{d x, d y\}$.

Como o fluido está continuamente em movimento, é utilizado um procedimento para identificar a região contendo fluido e a superfície livre. Observe a Figura 2.

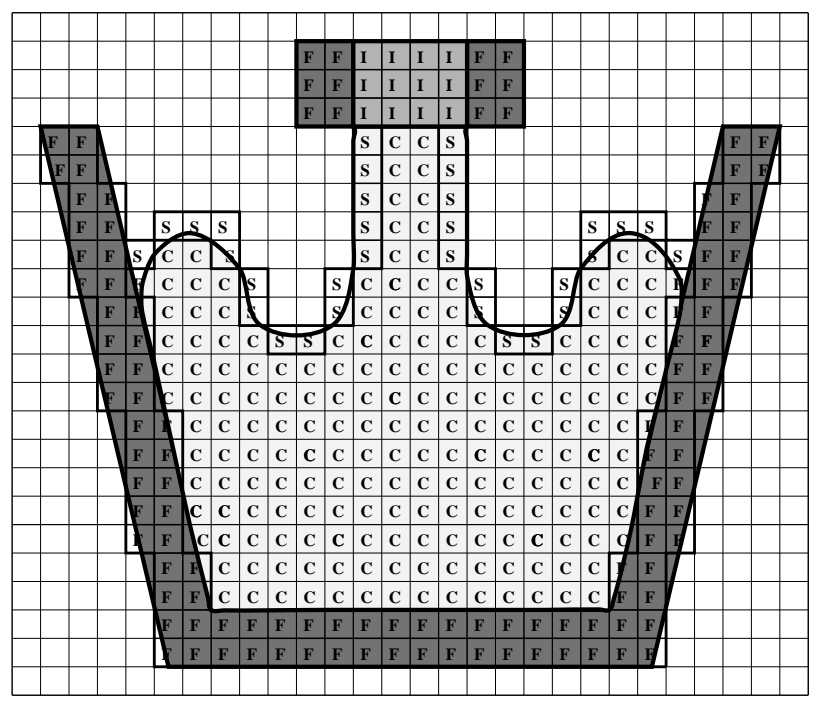

Figura 2: Domínio, malha e células

As células da malha tendo metade ou mais de seu volume contido no contorno rígido são classificadas como células FRONTEIRA (F); as células contendo a metade ou mais de seu volume em um injetor (células que representam a entrada do fluido na região de domínio do fluido) como INJETOR (I); e as células contendo a metade ou mais de seu volume em um ejetor (células que representam a saída do fluido na região de domínio do fluido) como EJETOR (E). As células que contém alguma porção da superfície do fluido (células que contém fluido e tem pelo menos uma 
adjacência com uma célula do tipo $\mathrm{V}$, essas células contém a superfície livre) são classificadas como células SUPERFÍCIE (S); as células que não contém fluido como VAZIA (V); e as células que contém fluido e não têm contato com células do tipo V como CHEIA (C). Na Figura 2, as células VAZIA (V) não apresentam o símbolo $\mathrm{V}$ para tornar a figura mais limpa.

Durante a execução de um certo problema, uma célula do tipo C pode tornar-se $\mathrm{S}$ e então $\mathrm{V}$, e vice-versa.

\section{Visualizador}

Os resultados da simulação de escoamentos de fluidos podem ser visualizados graficamente através do módulo de visualização Visflow-2D. Os resultados gerados pelo simulador são dados sobre o modelo de escoamento, a representação dos objetos geométricos e propriedades em instantes pré-definidos. O Visflow-2D permite a visualização gráfica do fluido e do domínio.

A entrada de dados do visualizador é feita por arquivos. O acesso aos dados do modelo que independem do tempo é feito por um arquivo e os que dependem do tempo é feito por uma série de arquivos, contendo informações sobre os objetos geométricos, propriedades, configuração das células, tempo e ciclo da simulação.

Dados de um determinado passo no tempo ficam armazenados em arquivos distintos, tornando, assim, o tempo de acesso a um dado do início da simulação igual ao acesso a outro no final da simulação e permitindo a visualização de campos mesmo antes do término da simulação.

O visualizador possui uma interface gráfica de fácil execução e as mesmas tarefas encontradas no modelador, além da visualização da pressão e das velocidades $u$ e $v$.

\section{Reiniciador}

O Resimflow-2D é o módulo responsável em reiniciar a simulação do ponto que parou ou em realizar modificações em algum campo para simular novamente. O reiniciador é importante também quando ocorre interrupção da execução do programa por algum motivo, como por exemplo, queda de energia elétrica.

O reiniciador possui uma interface gráfica de fácil execução. Os dados do modelo que podem ser alterados pelo Resimflow-2D são: ciclo final, tempo final, espaçamento de tempo para impressão e para gravação automática, tolerância para a solução da equação de Poisson e fatores de controle de passo.

\section{Comunicação entre os Módulos}

A comunicação entre os módulos do sistema é feita por arquivos. A saída do modelador é constituida de oito arquivos. O simulador tem como entrada oito arquivos com a mesma estrutura dos arquivos de saída do modelador tendo em vista que podem ter sido gerados pelo modelador, pelo próprio simulador, ou pelo reiniciador. 
Os arquivos de saída do simulador servem a quatro propósitos: para o final da simulação (a simulação pode ser continuada a partir destes dados), para o caso de ocorrência de algum erro durante a simulação, salvamento em tempos pré-definidos para o caso de interrupção da simulação por algum problema do sistema ou da máquina sendo utilizada (estes três propósitos têm os arquivos de saída com a mesma estrutura do modelador) e para a visualização dos resultados. Os arquivos de entrada do visualizador são os arquivos de saída do simulador que servem ao propósito de visualização dos resultados. Os arquivos de entrada do reiniciador podem ser uns dos três arquivos de saída do simulador cuja estrutura seja a mesma dos arquivos de saída do modelador e os arquivos de saída são, exatamente, os mesmos do modelador.

\section{Resultados}

\section{1- Splashing}

Este modelo possui um contêiner do tipo Box reto e um injetor. Os dados que configuram o escoamento são:

- domínio: $x \min =0.0, x \max =0.053$, ymin $=0.0$, ymax $=0.0565$;

- células: $i \max =106, j \max =113$;

- dimensão das células: $d x=0.0005, d y=0.0005$;

- tempo inicial: 0.0, tempo final: 1.0;

- ciclo inicial: 0, ciclo final: 50000;

- escala de comprimento em metros: 0.005;

- escala de velocidade em metros por segundos: 5.0;

- campo gravitacional: $g_{x}=0.0, g_{y}=-9.81$;

- viscosidade: 0.001;

- número de Reynolds: 25.0;

- número de Frouden: 22.576182;

- número de Strouhal: 1000.0;

- tolerância para a solução da equação de Poisson: 0.000001.
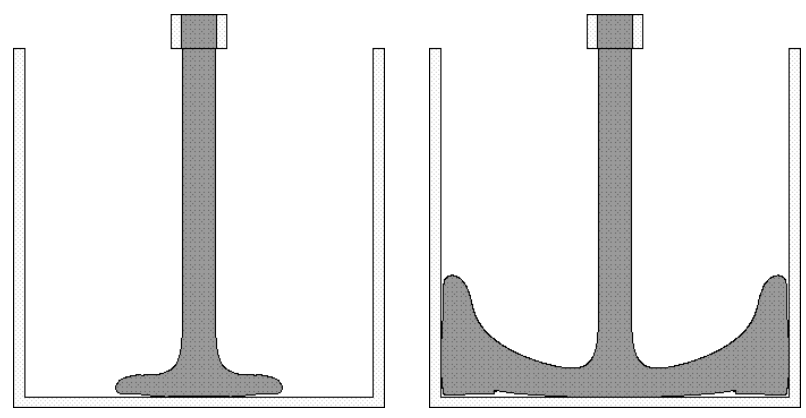

Figura 3: $t=0.0125$ e $t=0.025$ 

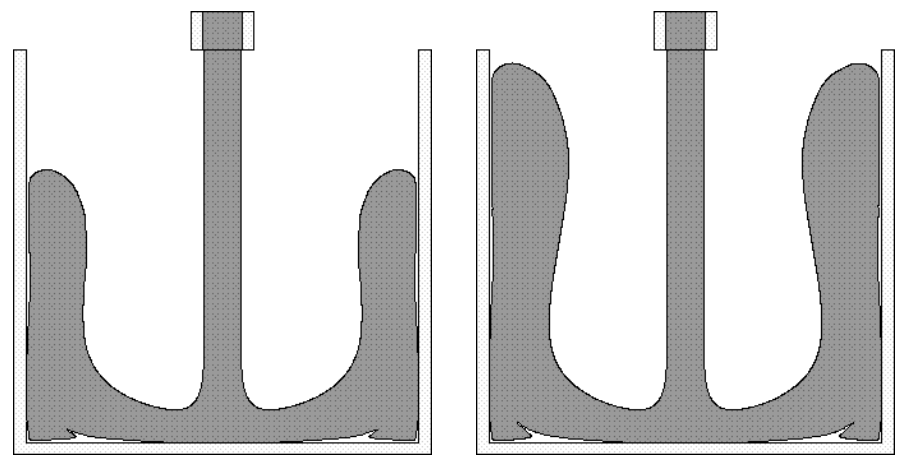

Figura 4: $t=0.0375$ e $t=0.05$

Em seguida, tem-se a pressão e a velocidade em x e em y deste modelo no tempo $t=0.05$.

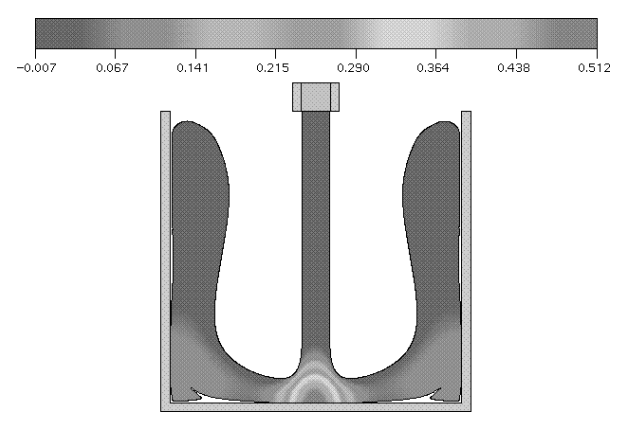

Figura 5: Pressão

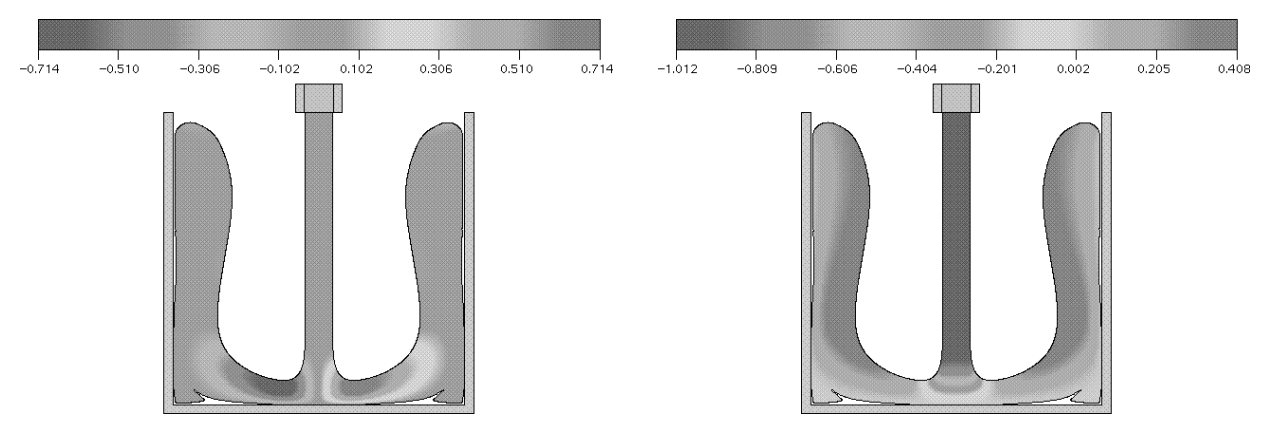

Figura 6: Velocidade em x e em y 


\section{2- Buckling Jet}

Este modelo possui um contêiner do tipo Box reto e um injetor. Os dados que configuram o escoamento são:

- domínio: $x \min =0.0, x \max =0.053$, ymin $=0.0$, ymax $=0.0565$;

- células: $i \max =106, j \max =113$;

- dimensão das células: $d x=0.0005, d y=0.0005$;

- tempo inicial: 0.0, tempo final: 1.0;

- ciclo inicial: 0, ciclo final: 300000;

- escala de comprimento em metros: 0.003;

- escala de velocidade em metros por segundos: 0.5;

- campo gravitacional: $g_{x}=0.0, g_{y}=-9.81$;

- viscosidade: 0.01;

- número de Reynolds: 0.015;

- número de Frouden: 2.9145726;

- número de Strouhal: 166,666667;

- tolerância para a solução da equação de Poisson: 0.0000001.

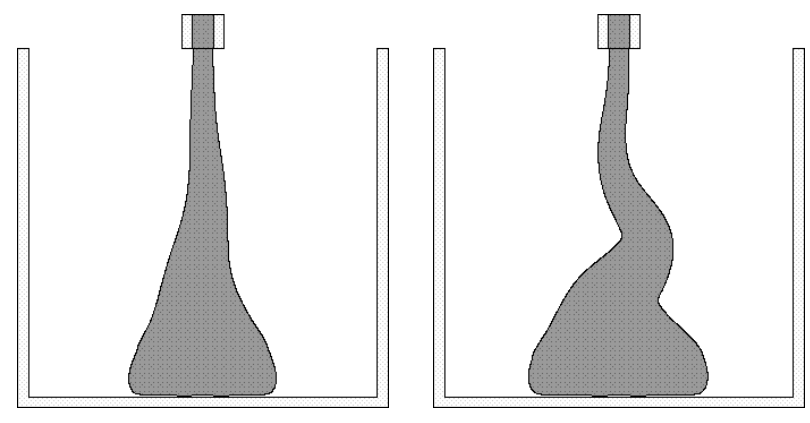

Figura $7: t=0.325$ e $t=0.375$
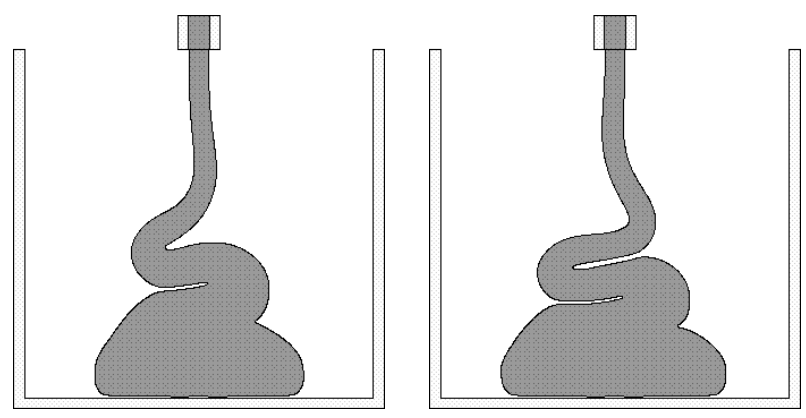

Figura 8: $t=0.425$ e $t=0.45$ 

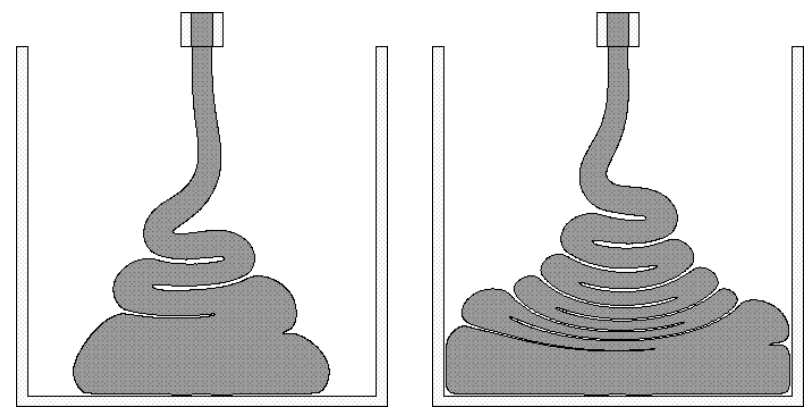

Figura 9: $t=0.5$ e $t=0.7$

\section{3- Steady Filling}

Este modelo possui um contêiner do tipo Box-Cilinder e um injetor. Os dados que configuram o escoamento são:

- domínio: $x \min =0.0, x \max =0.05, y \min =0.0, y \max =0.06$;

- células: $i \max =50$, jmax $=60$;

- dimensão das células: $d x=0.001, d y=0.001$;

- tempo inicial: 0.0, tempo final: 1.0;

- ciclo inicial: 0, ciclo final: 25000;

- escala de comprimento em metros: 0.004;

- escala de velocidade em metros por segundos: 0.5;

- campo gravitacional: $g_{x}=0.0, g_{y}=0.0$;

- viscosidade: 0.001;

- número de Reynolds: 2.0;

- número de Frouden: 2.5240939;

- número de Strouhal: 125.0;

- tolerância para a solução da equação de Poisson: 0.000001.
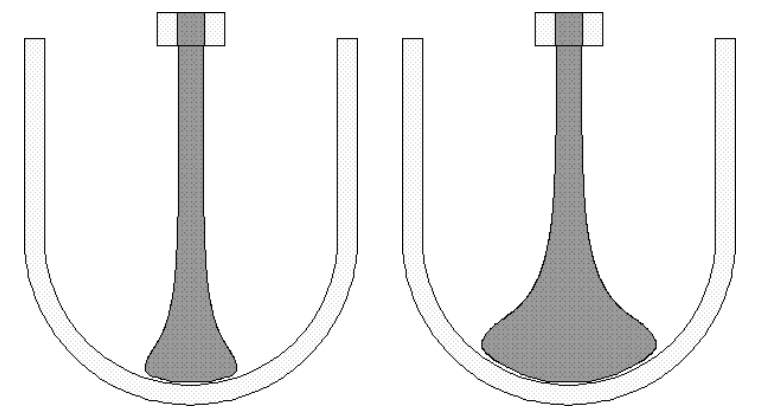

Figura 10: $t=0.15$ e $t=0.25$ 

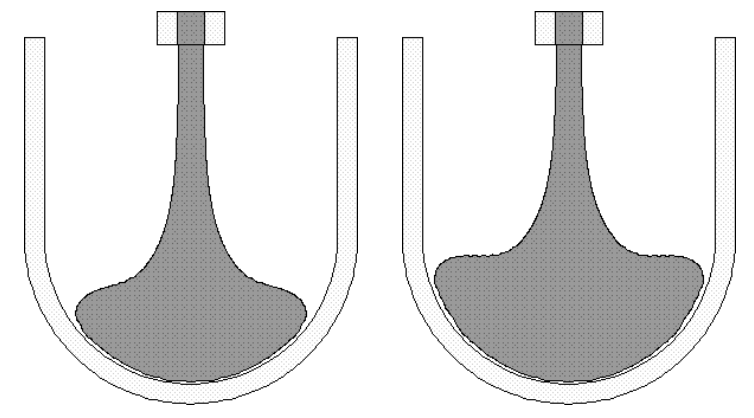

Figura 11: $t=0.35$ e $t=0.45$
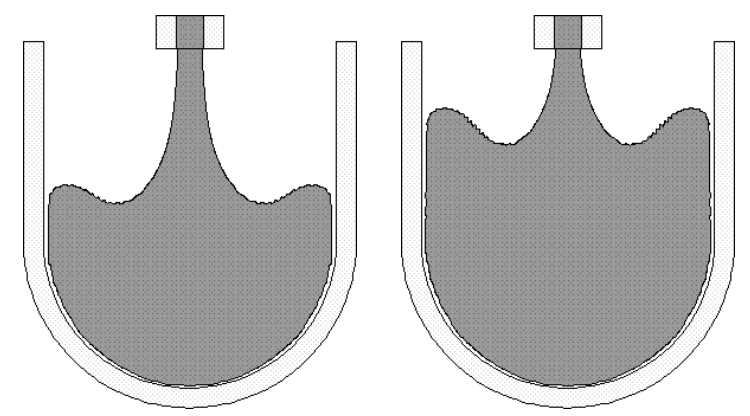

Figura 12: $t=0.65$ e $t=0.85$

\section{Conclusão}

Neste trabalho, foi apresentado o Freeflow-2D, um ambiente integrado para simulação de escoamentos bidimensionais incompressíveis com superfícies livres. Foi descrito os quatro módulos que compõem o sistema: Modflow-2D, Simflow-2D, Visflow-2D e Resimflow-2D. Foi explicado a estrutura de dados halfedge-2d, a comunicação entre os módulos e foram apresentados, como exemplos, três modelos simulados pelo Freeflow-2D.

Conclue-se, assim, que o Freeflow-2D é um sistema que atingiu seu objetivo de modelar, simular e visualizar escoamentos com a mesma precisão que o Freeflow, porém com uma estrutura de dados que permite extensões, além de contribuir como teste para extensões que se pensa em realizar no Freeflow-3D.

\section{Referências}

[1] F.H. Harlow and J.E. Welch, The MAC method, Phys. Fluids, 8 (1965), 2182; J.E. Welch, F.H. Harlow, J.P. Shannon, J.B. Daly, Los Alamos Scientific Labo- 
ratory, Report LA-3425, Los Alamos, NM, 1996.

[2] A. Amsden and F.H. Harlow, The SMAC method: A numerical technique for calculating incompressible fluid flows. Los Alamos Scientific Laboratory Report LA-4370, Los Alamos, NM, 1970.

[3] M.F. Tomé and S. McKee, GENSMAC: A computational marker-and-cell method for free surface flows in general domains, J. Comp. Phys., 110 (1994), 171-186.

[4] M.F. Tomé, B. Duffy and S. McKee, A numerical method for solving unsteady non-Newtonian free surface flows, Journal of Non-Newtonian Fluid Mechanics, 62 (1996), 9-34.

[5] J. Murakami, Desenvolvimento de um modelador de moldes e de um visualizador de escoamentos de fluidos. Dissertação de Mestrado, Instituto de Ciências Matemáticas de São Carlos, Universidade de São Paulo. São Carlos, 1996.

[6] A.C. Filho, J.A. Cuminato, M.C.F. Oliveira, R.D. Santi, M.F. Tomé e C.N.L. César, Freeflow-3D: um sistema integrado para simulação de escoamentos incompressíveis com superfícies livres (submetido).

[7] M.F. Tomé, A.C. Filho, J.A. Cuminato and S. McKee, GENSMAC-3D: implementation of the Navier-Stokes equation for 3D free surface flows. Notas do ICMSC-USP, ICMSC-USP, São Carlos, 1996.

[8] J. Oliveira, Desenvolvimento de um sistema de simulação de escoamentos de fluidos com superfícies livres bidimensionais. Dissertação de Mestrado, Instituto de Ciências Matemáticas de São Carlos, Universidade de São Paulo, São Carlos, 1999. 